\title{
Hearing loss and cognition in the Busselton Baby Boomer cohort: an epidemiological study
}

*Romola S. Bucks, $\mathrm{PhD}^{1}$, Patrick D. Dunlop, $\mathrm{PhD}^{1}$, Dunay Schmulian Taljaard, $\mathrm{PhD}{ }^{2,3,4}$

Christopher G. Brennan-Jones, BSc (Hons) ${ }^{3,4}$ Michael Hunter, $\mathrm{PhD}^{5,6}$ Keith Wesnes, PhD 7,8,9, Robert H. Eikelboom, $\mathrm{PhD}^{3,4,10}$

1. School of Psychology, University of Western Australia, Crawley, Australia

2. Audiology Department, Princess Margaret Hospital, Subiaco, Australia

3. Ear Sciences Centre, School of Surgery, University of Western Australia, Crawley, Australia

4. Ear Science Institute Australia, Subiaco, Australia

5. Busselton Population Medical Research Institute, Busselton, Australia

6. School of Population Health, University of Western Australia, Nedlands, Australia

7. Wesnes Cognition Ltd, Streatley on Thames, United Kingdom

8. Department of Psychology, University of Northumbria, Newcastle, United Kingdom

9. Centre for Human Psychopharmacology, Swinburne University, Melbourne, Australia

10. Department of Speech-Language Pathology and Audiology, University of Pretoria, Pretoria, South Africa

The work was conducted at the Busselton Health Study (MH), the Department of Psychology, UWA (RSB, PD), and the Ear Science Institute Australia (CB-J, DST, RE)

Short running title: Hearing loss and cognition in Baby Boomers 


\section{Funding:}

This work was supported by the Office of Science and the Department of Health of the Government of Western Australia, the Shire of Busselton, Ear Science Institute Australia Inc., Lions Hearing Foundation of Western Australia Inc., and private donations.

\section{Conflict of interest disclosures:}

Dr. Bucks has nothing to disclose. Dr. Dunlop has nothing to disclose. Dr. Schmulian Taljaard has nothing to disclose. Mr. Brennan-Jones reports grants from Cochlear Foundation Ltd and the Lions Hearing Foundation of Western Australia, outside the submitted work. Dr Hunter has nothing to disclose. Dr Wesnes was until February 2014 an employee of Bracket Global who provide the CDR System as a service in clinical trials. He currently runs a consultancy, which provides services concerning appropriate testing of cognitive function in clinical trials. Dr. Eikelboom reports non-financial support from Sonova Holdings Switzerland, non-financial support from Med-el, Austria, grants from Med-el, Austria, personal fees and non-financial support from National Health and Medical Research Council, Australia, outside the submitted work.

Word count: 2988 ( $\max 3000$, plus 43 references, $<=8$ tables with $<=6$ columns each) 250 word abstract

Corresponding author: Romola S. Bucks, PhD, School of Psychology (M304), University of Western Australia, 35 Stirling Highway, Crawley, WA 6009, Australia (romola.bucks@uwa.edu.au). 
Objective: To determine the relationship between peripheral hearing loss in Baby Boomers (better-ear measure) and cognitive function, taking into account the impact of depression or cognitive reserve on this relationship, and exploring binaural hearing.

Study Design: A prospective, epidemiology study.

Methods: Data from 1969 participants aged 45-66 years were collected in the Busselton Healthy Ageing Study. Participants were assessed using pure-tone air-conduction thresholds at octave frequencies $(250,500,1000,2000,4000$ and $8000 \mathrm{~Hz})$. Hearing loss (HL) was grouped using (i) pure-tone averages at $500-4000 \mathrm{~Hz}$ in the better-ear (BE4FA), or (ii) latent profile analysis (LPA) using all thresholds from both ears. Cognition was tested with the Cognitive Drug Research System, verbal-fluency, and National Adult Reading Test (premorbid-IQ). Regression was used to determine the impact of HL relative to no HL, on age and education-adjusted cognition, controlling for mood, sex, and premorbid-IQ.

Results: According to (i) BE4FA, 4.7\% had Mild (26-40dB), 0.8\% Moderate (41-60dB) and $0.3 \%$ Severe (61-80dB) HL. Based on (ii) the LPA, 20.5\% had high-frequency HL, $7.8 \%$ mid-to-high frequency, and 1.9\% significant HL across all frequencies. HL-Group was not a predictor of cognitive performance in any domain using BE4FA, and explained just $0.5 \%$ and $0.4 \%$ of variance in continuity-of-attention and speed-of-memory-retrieval using LPA. Critically, those with the worst hearing did not differ cognitively from those with the best.

Conclusions: Hearing loss is not an important determinant of contemporaneous attention, memory or executive function in middle-aged adults, once age, education, depression, cognitive reserve, and sex are controlled. 
Keywords: hearing impairment, cognition, epidemiology, ageing

Level of evidence: $2 \mathrm{a}$ 


\section{Introduction}

Hearing loss is a common disability, affecting over 360 million people globally ${ }^{1}$. Its prevalence and severity is higher amongst men and older adults ${ }^{2}$. In addition to reductions in quality of life ${ }^{3,4}$ and mood ${ }^{5}$, hearing loss has also been associated with loss of cognitive function in adults over the age of 60 . Studies reveal poorer cognition in those with poorer hearing, especially in memory and executive function ${ }^{6-8}$, faster decline in cognition in adults aged $55+$ years ${ }^{9}$, and increased risk of dementia ${ }^{10,11}$.

Proposed mechanisms are 1) hearing loss and cognition may change due to shared pathology (e.g. ageing, dementia), or 2) attentional or short-term/working memory resources are required to compensate for auditory processing deficits, leading to secondary, higherorder cognitive problems ${ }^{12,13}$. Alternatively, social isolation, due to impairment in hearing, may be a risk for cognitive decline/dementia, perhaps through lack of mental stimulation ${ }^{14,15}$.

However, three theoretical and methodological issues preclude the formation of a justifiable working hypothesis. First, hearing loss is associated with depression ${ }^{16}$, and depression is, in turn, a risk factor for poorer cognition ${ }^{17}$. Yet, few studies consider the role of depression in the relationship between hearing loss and cognition. Second, studies fail to control for the possibility that premorbid ability, or "cognitive reserve", may 'buffer' the effect of neurocognitive trauma ${ }^{18}$, and, thus, dilute the impact of hearing on cognitive function. Third, typically, significant hearing loss is indicated by a speech-frequency pure tone average (PTA in $\mathrm{dB}$ ) of air-conduction thresholds at 500 to $4000 \mathrm{~Hz}$ of over $25 \mathrm{~dB}$ in the better ear ${ }^{2,11}$. If one of the mechanisms by which cognition is impacted by hearing loss is via increased auditory processing demands, an index of hearing loss that includes the poorer ear may be a better index when exploring the relationship with cognition, than PTA from the better ear: hearing is a binaural, not a monaural, process. 
This paper reports data from the first 1969 participants assessed in the Busselton Healthy Ageing Study (BHAS). It explores the relationship between hearing impairment and cognition using a better-ear, pure tone average (PTA) as well as hearing-impairment profiles based on both ears. Hearing loss prevalence increases markedly from age 50 years ${ }^{2}$, whereas cognitive decline is evident as young as $45-49$ (3.6\% decline in reasoning over 10 years) ${ }^{19}$. Thus, a study of the relationship between hearing and cognition in middle-aged adults is of particular relevance. In the event that the two are causally related, it will be at around this age that interventions to improve cognition and stave off dementia may have their greatest impact ${ }^{20}$. We hypothesised that greater hearing loss would be associated with poorer cognitive performance after controlling for cognitive reserve (premorbid-IQ), age, education, depression, and sex.

\section{Materials and Methods}

The BHAS is a longitudinal study of community-dwelling adults, born 1946 to 1964 , randomly selected from the Busselton Shire, Western Australia, electoral roll. Participants completed questionnaires detailing demographics, medical history, physical symptoms and quality of life, including a measure of depression (PHQ-9) ${ }^{21}$, and the question "Do you have a hearing impairment?". Participants then attended the Busselton study centre for 4 hours to complete comprehensive physical and cognitive assessments. Clinically relevant results were provided to all participants. The University of Western Australia Human Research Ethics Committee approved the study (Number RA/4/1/2203) and all participants gave written, informed consent. See James et al., 2013 for the full protocol ${ }^{22}$.

Between May 2010 and June 2012, 2023 participants (66.3\% of those approached) were recruited. Data from 27 were incomplete/had unreliable hearing tests ${ }^{23}$, and 27 did not complete cognitive testing, leaving 1969 (46.2\% male) aged 45-66 years $(M=56.2, \mathrm{SD}=5.5$ 
years) of whom 41 wore hearing aids (unilateral hearing aid $n=10$; bilateral hearing aids $n=$ $31)$.

Hearing thresholds

After screening for contraindications to hearing assessment by otoscopy and tympanometry, pure tone air conduction thresholds at octave frequencies of 250,500, 1000, 2000, 4000 and $8000 \mathrm{~Hz}$ were obtained with an AMTAS automated audiometer (Audiometry Incorporated, USA) ${ }^{24}$ in a sound treated booth. See ${ }^{22}$ for details.

Hearing was classified using: (i) 4 frequency average across 500, 1000, 2000 and 4000 $\mathrm{Hz}$ for each ear, as normal, mild, moderate, severe or profound based on the PTA of the better ear $^{1}$; (ii) empirically, via Latent Profile Analysis ${ }^{25}$.

\section{Cognition}

The Cognitive Drug Research (CDR) ${ }^{26}$ computerised assessment System (Bracket Global, UK) is sensitive to subtle cognitive changes, and has the largest normative database of its kind ${ }^{26}$. The battery assesses: immediate and delayed word recall and recognition, simple and choice reaction time, digit vigilance, spatial and numeric working memory, and delayed picture recognition. Participants also completed verbal fluency (letters C, F, L) as a measure of generative executive function ${ }^{27}$. Pre-morbid-IQ was estimated from the National Adult Reading Test (NART-2 ${ }^{28}$ ) and used as an index of cognitive reserve. This is an accepted method of estimating premorbid IQ from current capacity, by testing reading of irregular nouns. ${ }^{28}$ The number of errors is used to estimate IQ, with fewer errors being associated with higher premorbid IQ. Irregular word reading ability has been shown both to correlate highly with full scale $\mathrm{IQ}^{28}$ and to be resistant to decline due to ageing or dementia. ${ }^{29}$ Participants, who attended with hearing aid(s), were instructed to wear them during testing. 
Cognitive outcome measures: The various task measures from the CDR System were collapsed into cognitive scores derived by factor analysis ${ }^{30}$ : Quality of Episodic Secondary Memory, Quality of Working Memory, Speed of Memory, Continuity of Attention, and Power of Attention.

\section{Results}

As expected, older age and higher premorbid-IQ were associated with lower and higher scores, respectively, on all cognitive measures (Table 1). Depression scores (from the PHQ-9) and female sex were associated with poorer fluency and better continuity of attention, respectively, and female sex was associated with better quality of episodic memory (Table 1), confirming the need to control for age and education, premorbid-IQ and depression in all inferential analyses, since such covariates may mask effects of hearing loss on cognition. Thus, age, education, premorbid-IQ and depression were treated as covariates in all regression analyses. See Tables 2 and 3 for raw means \pm SD by hearing groups.

To test the central hypothesis that cognitive performance is negatively associated with hearing loss, we undertook two sets of analyses: (i) a classification based on four frequency average in the better ear (BE4FA); (ii) Latent Profile Analysis ${ }^{25}$ to classify individuals based on their hearing thresholds across all six frequencies in both ears. Both methods are described below and are compared with self-reported hearing loss. We then investigated the relationships between hearing loss and performance on the cognitive assessments, controlling for confounds.

Standard Hearing Loss Severity Classification (Standard HL Severity Groups) Participants were classified into 1 of 4 groups, based on four-frequency average: the mean hearing threshold (in dB) across $500 \mathrm{~Hz}, 1000 \mathrm{~Hz}, 2000 \mathrm{~Hz}$, and $4000 \mathrm{~Hz}$ in the better ear (BE4FA). Participants were classified into four Standard HL Severity groups according to 
Table 1.

Means and Standard Deviations of, and Intercorrelations among, Study Variables

\begin{tabular}{|c|c|c|c|c|c|c|c|c|c|c|c|}
\hline Variable & Mean & SD & 1 & 2 & 3 & 4 & 5 & 6 & 7 & 8 & 9 \\
\hline 1. Phonemic Verbal Fluency (words) & 30.86 & 7.20 & & & & & & & & & \\
\hline 2. Continuity of Attention (max. 100) & 90.78 & 3.89 & .11 & & & & & & & & \\
\hline 3. Speed of Attention (ms) & 1238.46 & 136.65 & -.27 & .01 & & & & & & & \\
\hline 4. Quality of Episodic Memory (max. 400) & 190.27 & 43.26 & .29 & .15 & -.21 & & & & & & \\
\hline 5. Quality of Working Memory (max. 200) & 188.10 & 15.34 & .15 & .19 & -.08 & .16 & & & & & \\
\hline 6. Speed of Memory Retrieval (ms) & 4240.12 & 862.72 & -.29 & -.11 & .43 & -.34 & -.16 & & & & \\
\hline 7. Sex & & & .18 & .06 & -.03 & .23 & .03 & -.04 & & & \\
\hline 8. Age & 56.25 & 5.53 & -.21 & -.15 & .20 & -.21 & -.09 & .21 & -.04 & & \\
\hline 9. NART-IQ (premorbid-IQ) & 95.37 & 10.32 & -.44 & -.19 & .17 & -.22 & -.17 & .20 & -.13 & 0 & \\
\hline 10. PHQ9 Total Score (max. 27) & 2.74 & 3.71 & .05 & -.09 & 0 & -.03 & -.03 & .01 & .09 & -.05 & 0 \\
\hline
\end{tabular}

Note. Bivariate $n$ ranges from 1937 to 1969 ; Sex is $0=$ male $1=$ female; For all $|\mathrm{r}| \geq .05, p<0.05$. For all $|\mathrm{r}| \geq .06, p<0.01$. 
Table 2 .

Normed Means and Standard Deviations on all Cognitive Performance Variables from each of the Standard HL Severity Groups (BE4FA)

\begin{tabular}{|c|c|c|c|c|c|c|c|}
\hline Hearing Loss Severity Group & & $\begin{array}{c}\text { Phonemic Verbal } \\
\text { Fluency } \\
\text { (words) }\end{array}$ & $\begin{array}{l}\text { Continuityof } \\
\text { Attention } \\
(\max .95)\end{array}$ & $\begin{array}{l}\text { Power of } \\
\text { Attention } \\
\quad(\mathrm{ms})\end{array}$ & $\begin{array}{c}\text { Quality of } \\
\text { Episodic Memory } \\
\text { (max. 400) }\end{array}$ & $\begin{array}{l}\text { Quality of } \\
\text { Working Memory } \\
\text { (max. } 2.0)\end{array}$ & $\begin{array}{c}\text { Speed of Memory } \\
\text { Retrieval } \\
(\mathrm{ms})\end{array}$ \\
\hline $\begin{array}{l}\text { Normal Hearing } \\
(\mathrm{n}=1838-1857)\end{array}$ & & $30.98(7.17)$ & $90.84(3.88)$ & $1237.37(136.54)$ & $184.35(46.40)$ & $1.88(0.15)$ & $4229.24(842.43)$ \\
\hline $\begin{array}{l}\text { Mild Hearing Loss } \\
(\mathrm{n}=91-92)\end{array}$ & & $28.81(7.59)$ & 90.08 (3.99) & $1250.53(138.89)$ & $172.26(48.73)$ & $1.87(0.15)$ & $4341.61(1109.51)$ \\
\hline $\begin{array}{l}\text { Moderate Hearing Loss } \\
(\mathrm{n}=15)\end{array}$ & & $29.53(8.08)$ & $89.07(3.71)$ & $1270.20(132.72)$ & $173.57(40.83)$ & $1.90(0.09)$ & $4590.19(873.76)$ \\
\hline \multirow{2}{*}{$\begin{array}{l}\text { Severe Hearing Loss } \\
(n=4-5)\end{array}$} & & $29.33(4.79)$ & $89.40(4.62)$ & $1323.24(139.36)$ & $161.00(35.05)$ & $1.88(0.10)$ & $5413.77(1924.62)$ \\
\hline & Total & $30.86(7.20)$ & $90.78(3.89)$ & $1238.46(136.65)$ & $183.65(46.51)$ & $1.88(0.15)$ & $4240.12(862.72)$ \\
\hline$F(3,1952-1963)$ & & 2.861 & 2.315 & 1.19 & 2.60 & .16 & 4.40 \\
\hline$p$ & & .036 & .074 & .312 & .051 & 0.926 & 0.004 \\
\hline$\eta_{\mathrm{p}}^{2}$ & & .004 & .004 & .002 & .004 & 0 & .007 \\
\hline
\end{tabular}

Note. Denominator degrees of freedom vary due to missing data on some cognitive measures. Higher scores on all measures indicate better (more accurate or faster) performance; $\eta_{\mathrm{p}}^{2}$ effect sizes, small $=0.01$; medium $=0.06$; large $=0.14^{50}$ 
Table 3.

Means and Standard Deviations on all Cognitive Performance Variables from the Latent Profiles

\begin{tabular}{|c|c|c|c|c|c|c|}
\hline & $\begin{array}{c}\text { Phonemic Verbal } \\
\text { Fluency } \\
\text { (words) }\end{array}$ & $\begin{array}{c}\text { Continuity of } \\
\text { Attention } \\
(\max .95)\end{array}$ & $\begin{array}{l}\text { Power of Attention } \\
\text { (ms) }\end{array}$ & $\begin{array}{l}\text { Quality of Episodic } \\
\text { Memory (max. 400) }\end{array}$ & $\begin{array}{l}\text { Quality of Working } \\
\text { Memory (max. 2.0) }\end{array}$ & $\begin{array}{c}\text { Speed of Memory } \\
\text { Retrieval } \\
\text { (ms) }\end{array}$ \\
\hline Profiles $1 \& 2(n=1375)$ & $31.50(7.21)$ & $91.17(3.41)$ & $1232.11(131.03)$ & $188.02(46.17)$ & $1.89(0.15)$ & $4204.14(851.08)$ \\
\hline Profile $3(n=403)$ & $29.61(6.81)$ & $89.99(4.90)$ & $1249.51(147.57)$ & $174.22(45.10)$ & $1.87(0.16)$ & $4235.82(742.27)$ \\
\hline Profile $4(n=153)$ & $28.87(7.57)$ & $89.79(4.26)$ & $1262.49(148.10)$ & $169.98(46.61)$ & $1.86(0.16)$ & $4568.11(1110.24)$ \\
\hline Profile $5(\mathrm{n}=38)$ & $29.03(6.04)$ & $89.26(4.09)$ & $1253.72(153.00)$ & $178.96(48.94)$ & $1.89(0.10)$ & $4255.06(1049.68)$ \\
\hline Total & $30.86(7.20)$ & $90.78(3.89)$ & $1238.46(136.65)$ & $183.65(46.51)$ & $1.88(0.15)$ & $4240.12(862.72)$ \\
\hline$F(3,1953-1963)$ & 1.58 & 15.71 & 3.62 & 14.30 & 1.58 & 8.31 \\
\hline$p$ & .193 & $<.001$ & .013 & $<.001$ & .193 & $<.001$ \\
\hline$\eta_{p}^{2}$ & .002 & .023 & .006 & .022 & .002 & .013 \\
\hline
\end{tabular}

Note. Denominator degrees of freedom vary slightly due to missing data on some cognitive measures. Higher scores on all measures indicate better (greater accuracy or faster) performance; $\eta_{p}^{2}$ effect sizes, small $=0.01$; medium $=0.06$; large $=0.14^{50}$ 
World Health Organisation Guiedelines $^{1}$ : (1) Normal - BE4FA at 25dB or less $(n=1857),(2)$ Mild - BE4FA between 26 and 40dB ( $n=92)$, (3) Moderate - BE4FA between 41 and $60 \mathrm{~dB}$ $(n=15),(4)$ Severe - BE4FA between 61 and $80 \mathrm{~dB}(n=5)$. No participants had Profound HL - BE4FA of $81 \mathrm{~dB}$ or over.

Next, two-step hierarchical regression analyses of the normed cognitive scores were undertaken. Covariates were entered in Step 1: Sex, NART-predicted IQ, Education (dummy coded), and PHQ-9 Total Scores. Dummy-coded Standard HL Severity Group variables were entered in Step 2. The parameters estimated represent the impact of being in 1 of the 3 nonNormal HL groups (Mild/Moderate/Severe), relative to the Normal Hearing group, after accounting for covariates (Table 4).

None of the cognitive variables was significantly predicted by HL Severity Group membership, after controlling for covariates. The failure to find an effect of HL may relate to the groups being too 'blunt' to act as powerful predictors, the measurement of HL using better-ear only, or the relatively small sizes of the Severe and Moderate HL Groups. Thus, Latent Profile Analysis was used to assign participants into hearing loss groups.

Latent Profile Analyses

Latent Profile Analysis (LPA) identifies latent (i.e. unobserved) groups (profiles) of people on the basis of scores on a set of indicator (i.e. observed) variables ${ }^{25}$. Profiles are determined that account for the variance shared amongst the indicator variables. Unlike cluster analysis, cases are probabilistically assigned to profiles. The first step in LPA is to determine the optimal number of profiles ${ }^{31}$. In deciding on the number of Profiles, we considered: (1) statistical fit, (2) pragmatic inspection of the nature of the ProfilesMuthén and Muthén ${ }^{32}$, and (3) inspection of the number in each Profile. 


\section{Table 4.}

Regression of Raw Cognitive Performance Variables on Covariates and Standard HL Severity Groups (BE4FA)

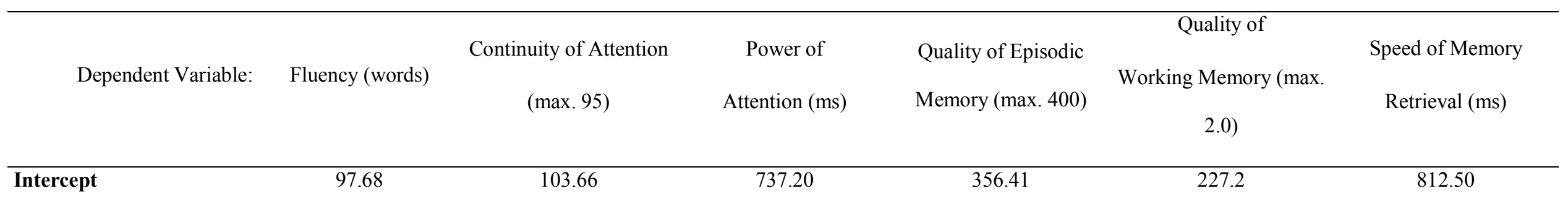

\begin{tabular}{|c|c|c|c|c|c|c|}
\hline Covariates: & & & & & & \\
\hline Sex $(0=$ Male, $1=$ Female $)$ & $2.70 * *(1.81,3.58)$ & $0.26(-0.07,0.59)$ & $2.44(-9.46,14.34)$ & $18.56^{* *}(14.63,22.50)$ & $0.17(-1.20,1.53)$ & $-10.16(-85.07,64.75)$ \\
\hline Age & $-0.26^{* *}(-0.34,-0.18)$ & $-0.11^{* *}(-0.14,-0.08)$ & $5.22 * *(4.14,6.29)$ & $-1.70 * *(-2.05,-1.35)$ & $-0.25 * *(-0.37,-0.13)$ & $32.35^{* *}(25.60,39.10)$ \\
\hline Educ. $=$ Primary School ${ }^{\mathrm{a}}$ & $2.72(-0.72,6.16)$ & $-0.72(-2.00,0.56)$ & $-29.16(-75.30,16.97)$ & $-3.82(-19.05,11.42)$ & $0.29(-5.00,5.58)$ & $29.79(-260.45,320.02)$ \\
\hline Educ. $=$ High School ${ }^{\mathrm{a}}$ & $0.51(-0.66,1.67)$ & $-0.32(-0.75,0.12)$ & $-10.68(-26.33,4.97)$ & $-0.77(-5.94,4.41)$ & $-0.09(-1.88,1.71)$ & $79.54(-19.00,178.09)$ \\
\hline Educ. $=$ Technical College ${ }^{a}$ & $0.16(-1.12,1.44)$ & $-0.29(-0.76,0.19)$ & $-6.91(-24.09,10.27)$ & $-0.02(-5.70,5.66)$ & $0.27(-1.70,2.24)$ & $44.74(-63.42,152.90)$ \\
\hline NART-IQ & $-0.50 * *(-0.54,-0.46)$ & $-0.07 * *(-0.08,-0.05)$ & $2.24 * *(1.66,2.81)$ & $-0.89 * *(-1.08,-0.70)$ & $-0.26^{* *}(-0.32,-0.19)$ & $16.18^{* *}(12.56,19.79)$ \\
\hline PHQ 9 Total Score & $0.10(-0.02,0.22)$ & $-0.10 * *(-0.14,-0.06)$ & $0.28(-1.30,1.87)$ & $-0.69 *(-1.21,-0.16)$ & $-0.14(-0.32,0.04)$ & $5.54(-4.45,15.52)$ \\
\hline HL Severity Group: & & & & & & \\
\hline Mild Hearing Loss ${ }^{\mathrm{b}}$ & $-1.83(-3.92,0.26)$ & $-0.24(-1.01,0.54)$ & $-7.45(-35.46,20.56)$ & $-0.01(-0.20,0.17)$ & $0.32(-2.89,3.54)$ & $-31.40(-207.59,144.79)$ \\
\hline
\end{tabular}


Moderate Hearing Loss ${ }^{\mathrm{b}}$

Severe Hearing Loss ${ }^{\mathrm{b}}$
$2.77(-2.40,7.93)$

$-3.81(-12.42,4.81)$

\begin{abstract}
$-0.79(-2.71,1.13)$
\end{abstract}
$-0.79(-3.99,2.41)$
$-2.28(-71.60,67.03)$

$47.61(-67.97,163.18)$
$0.04(-0.42,0.50)$

$-0.33(-1.10,0.44)$
$4.28(-3.67,12.23)$

$2.20(-11.05,15.46)$
$171.72(-264.33,607.78)$

$951.31 *(224.28$

1678.33)

\begin{tabular}{|c|c|c|c|c|c|c|}
\hline Overall $R^{2}$ & .258 & .073 & .074 & .133 & .040 & .087 \\
\hline$F$-test $(10,1933-1940)$ & $67.08 * *$ & $15.15^{* *}$ & $15.40 * *$ & $29.66 * *$ & $8.00 * *$ & $18.43 * *$ \\
\hline Categories added) & & & & & & \\
\hline$\Delta R^{2} F$-test $(3,1933-1940)$ & 1.62 & 0.40 & 0.31 & 0.18 & 0.36 & 2.44 \\
\hline
\end{tabular}

Note. ${ }^{*} p<0.05,{ }^{* *} p<0.01 .{ }^{a}$ Reference category is Highest Education $=$ University Degree. Educ. $=$ Highest Education; ${ }^{b}$ Normal Hearing is the

reference HL Severity Group. HL = Hearing Loss. Denominator degrees of freedom vary due to missing data on some cognitive measures. Higher scores on all measures indicate better (more accurate or faster) performance. Unless otherwise stated, figures in cells are unstandardised regression coefficients, and figures in parentheses are the bounds of the $95 \%$ confidence intervals. 


\section{Table 5.}

Fit Indices for 1 Latent Profile to 6 Latent Profile Models

\begin{tabular}{|c|c|c|c|c|c|}
\hline \multirow[b]{2}{*}{ Number of } & \multicolumn{5}{|c|}{$\triangle \mathrm{BIC}$} \\
\hline & & BIC (sample & (relative to & LMRLRT & BLRT \\
\hline Profiles & AIC & size adjusted) & K-1 Profiles) & (p-value) & (p-value) \\
\hline 1 & 192745.3 & 192782.5 & -- & -- & -- \\
\hline 2 & 186446.0 & 186504.9 & -3156.7 & 0.006 & $<0.001$ \\
\hline 3 & 183926.1 & 184006.7 & -2498.1 & 0.711 & $<0.001$ \\
\hline 4 & 182337.6 & 182439.9 & -1566.8 & 0.234 & $<0.001$ \\
\hline 5 & 180988.6 & 181112.6 & -1327.3 & 0.107 & $<0.001$ \\
\hline 6 & 180377.7 & 180523.5 & -589.1 & 0.462 & $<0.001$ \\
\hline
\end{tabular}

Note. $\mathrm{AIC}=$ Akaike Information Criterion; $\mathrm{BIC}=$ Bayesian Information Criterion; lower AIC and BIC indices indicate better fit; $\Delta \mathrm{BIC}=$ change in

BIC as number of profiles increases; LMRLRT $=$ Lo-Mendell-Rubin Likelihood Ratio Test - a smaller $p$ value suggests that the model with $k$ profiles is a better fit to k-1 profiles; BLRT = Bootstrap Likelihood Ratio Test - a significant $\mathrm{p}$ value indicates that the model with $\mathrm{k}-1$ profiles is a better fit to $\mathrm{k}$ profiles. 
The indicators were all hearing thresholds in both ears. Since ears are 'nested' within an individual, a two-level LPA was undertaken in MPlus 7.2, selecting 500 from a set of 10000 random starting values ${ }^{33}$. Table 5 gives fit statistics (AIC and BIC) of models with 1 through 6 profiles, and statistical tests of the comparison of a model with $\mathrm{K}$ and $\mathrm{K}-1$ profiles (the p-values for the Lo-Mendall-Rubin Likelihood Ratio test [LMRLRT], and the Bootstrapped Likelihood Ratio Test [BLRT] $)^{31}$. As can be seen, the fit statistics do not clearly indicate the optimal number of profiles. A decline in the magnitude of the reduction of the BIC as the number of profiles increases is observed, but the LMRLRT and BLRT return contrary advice: the former suggests a two profile solution is preferable, whereas the BLRT suggests as many as seven profiles may be needed.

Common to all of the models was a distinct (and usually the largest) profile of individuals with good hearing across all frequencies, and a relatively small profile comprising individuals with significant hearing impairment. Further, in all models, there were no marked differences in thresholds between the left and right ears. After consideration, we retained the 5-profile model (for brevity, we reproduce only the 5 profile solution here, but Figures for 3, 4, and 6 profile solutions are available on request): Profile $1(n=1117)$ "Excellent hearing" sensitive to sounds lower than the $20 \mathrm{~dB}$ 'normal' hearing criterion; Profile $2(\mathrm{n}=258)$ "Normal hearing" - sensitive to sounds approximately at $20 \mathrm{~dB}$ 'normal' hearing criterion; Profile $3(\mathrm{n}=403)$ "High frequency impaired" - excellent to normal hearing up to $2000 \mathrm{~Hz}$, but a sharp decline at higher frequencies; Profile $4(\mathrm{n}=153)$ "Mid-to-high frequency impaired" - normal hearing up to $1000 \mathrm{~Hz}$, but a significant decline at higher frequencies; and Profile $5(\mathrm{n}=38)$ "Significant impairment" - decreased sensitivity to all frequencies. The entropy statistic (range 0-1), indicates confidence in the classifications of participants into their respective profiles. For the five profile model, entropy was 0.917 , and thus for analyses that followed, we classified participants into their most likely profile. 


\section{Table 6.}

Comparison of the Latent Profiles against a) the question "Do you have a hearing impairment?" and b) Standard HL Severity Groups

\section{Do you have a hearing}

impairment?

\begin{tabular}{|c|c|c|c|c|c|c|}
\hline & & & Normal & Mild Hearing & Moderate & Severe \\
\hline Profile Number & No & Yes & Hearing & Loss & Hearing Loss & Hearing Loss \\
\hline $1 \& 2$ combined - "Normal hearing" & $92.0 \%$ & $8.0 \%$ & $99.5 \%$ & $0.5 \%$ & 0 & 0 \\
\hline 3 - "High frequency impaired" & $70.4 \%$ & $29.6 \%$ & $100.0 \%$ & 0 & 0 & 0 \\
\hline 4 - "Mid-to-high frequency impaired" & $38.3 \%$ & $61.7 \%$ & $50.0 \%$ & $46.1 \%$ & $3.9 \%$ & 0 \\
\hline 5 - "Significant impairment" & $21.1 \%$ & $78.9 \%$ & $26.3 \%$ & $36.8 \%$ & $23.7 \%$ & $13.2 \%$ \\
\hline Total & $82.0 \%$ & $18.0 \%$ & $94.3 \%$ & $4.7 \%$ & $0.8 \%$ & $0.3 \%$ \\
\hline
\end{tabular}

Note. Percentages are within-Profile for the whole sample

\section{Standard HL Severity Groups}


The approach to deriving profiles of hearing loss via clustering methods is relatively novel. Only one other study has used statistical methods (k-means cluster analysis) to categorise participants based on bilateral air-conduction audiograms in 20-69 year olds ( $\mathrm{see}^{34}$ ) but it did not explore the relationship to other variables. Thus, to ensure that the profiles represented differential levels of hearing impairment, we compared profile members on selfreported hearing-loss and against BE4FA classifications (Table 6). There was a clear association between self-reported hearing impairment and profile membership, $\chi^{2}(3, N=$ $1966)=421.6, p<.001$. There were inconsistencies, however, between the profiles and BE4FA categorisation, $\chi^{2}(3, \mathrm{~N}=1969)=1330.3, p<.001$. Nearly all members of Profiles 1 to 3 were defined as having normal hearing based on BE4FA, despite Profile 3 demonstrating hearing dysfunction at higher frequencies (Figure 1). Profile 4 tended to be diagnosed as suffering some form of hearing loss, with $<50 \%$ having Normal Hearing on BE4FA. Members of Profile 5 were most likely to have been diagnosed as having hearing loss, and were the only participants with Severe HL.

Although the five profile model was preferred, we combined members of Profiles 1 and 2 (those with Excellent or Normal hearing; Figure 1) into a single 'Normal Hearing' Profile, with $n=1375$, since no distinction is made clinically between them: both have normal hearing. This approach to combining conceptually similar but statistically distinct latent profiles is accepted practice, and did not appreciably affect the results of later analyses.

Predicting Cognitive Performance with the Latent Profiles Two-step regression analyses were undertaken as above (Table 7). Only Continuity of Attention and Speed of Memory Retrieval were significantly related to Profile membership after controlling for covariates. However, the variance explained was negligible $(0.5 \%$ and $0.4 \%$, respectively). For Continuity of Attention, members of the combined Profiles $1 \& 2$ 


\section{Table 7.}

Regression of Raw Cognitive Performance Variables on Controls and Profile Membership

\begin{tabular}{|c|c|c|c|c|c|c|}
\hline \multirow{4}{*}{ Dependent Variable: } & \multirow{4}{*}{ Fluency (words) } & Continuity of & & \multicolumn{3}{|c|}{ Quality of } \\
\hline & & & Speed of & Quality of Episodic & & Speed of Memory \\
\hline & & Attention & & & Working Memory & \\
\hline & & & Attention (ms) & Memory (max. 400) & $(20)$ & Retrieval (ms) \\
\hline Intercept & 97.89 & 102.93 & 730.37 & 357.10 & 227.13 & 786.10 \\
\hline
\end{tabular}

\begin{tabular}{|c|c|c|c|c|c|c|}
\hline \multicolumn{7}{|l|}{ Control Variables: } \\
\hline $\operatorname{Sex}(0=$ Male, $1=$ Female $)$ & $2.80 * *(1.86,3.73)$ & $0.13(-0.22,0.47)$ & $1.38(-11.12,13.88)$ & $18.65(14.51,22.78)$ & $0.17(-1.26,1.60)$ & $-26.68(-105.27,51.91)$ \\
\hline Age & $-0.27 * *(-0.35,-0.18)$ & $-0.10 * *(-0.13,-0.06)$ & $5.33 * *(4.21,6.46)$ & $-1.71 * *(-2.09,-1.34)$ & $-0.25^{* *}(-0.38,-0.12)$ & $32.78 * *(25.71,39.85)$ \\
\hline Educ. $=$ Primary School $^{\mathrm{a}}$ & $2.73(-0.72,6.17)$ & $-0.73(-2.01,0.54)$ & $-29.50(-75.65,16.66)$ & $-3.79 * *(-19.03,11.46)$ & $0.34(-4.95,5.64)$ & $14.60(-275.48,304.68)$ \\
\hline Educ. $=$ High School $^{\mathrm{a}}$ & $0.53(-0.64,1.70)$ & $-0.32(-0.75,0.12)$ & $-10.72(-26.37,4.94)$ & $-0.74(-5.92,4.44)$ & $-0.06(-1.85,1.74)$ & $77.49(-20.98,175.95)$ \\
\hline Educ. $=$ Technical College ${ }^{a}$ & $0.16(-1.13,1.44)$ & $-0.29(-0.77,0.18)$ & $-6.87(-24.05,10.31)$ & $-0.03(-5.71,5.65)$ & $0.29(-1.68,2.26)$ & $47.68(-60.38,155.74)$ \\
\hline NART-IQ & $-0.50 * *(-0.54,-0.46)$ & $-0.06 * *(-0.08,-0.05)$ & $2.26^{* *}(1.69,2.84)$ & $-0.89 * *(-1.08,-0.70)$ & $-0.26 *(-0.33,-0.19)$ & $16.49^{* *}(12.87,20.11)$ \\
\hline PHQ 9 Total Score & $0.10(-0.02,0.22)$ & $-0.10 * *(-0.14,-0.06)$ & $0.27(-1.32,1.85)$ & $-0.68 *(-1.21,-0.16)$ & $-0.14(-0.32,0.04)$ & $5.57(-4.39,15.53)$ \\
\hline \multicolumn{7}{|l|}{ Profile Membership: } \\
\hline Profile $3^{b}$ & $0.46(-0.73,1.64)$ & $-0.57 *(-1.01,-0.13)$ & $-5.28(-21.19,10.63)$ & $0.44(-4.83,5.71)$ & $0.27(-1.55,2.10)$ & $-117.33 *(-217.47$ \\
\hline
\end{tabular}


Profile $4^{\mathrm{b}}$

Profile $5^{\mathrm{b}}$
$-0.62(-2.36,1.12)$

$-0.48(-3.70,2.75)$

$-0.64(-1.29,0.00)$

$-5.36(-28.67,17.94)$

$-0.08(-7.78,7.62)$

$-1.22 *(-2.41,-0.03) \quad-9.44(-52.60,33.73)$

$0.27(-13.99,14.53)$

$-0.16(-2.83,2.51)$

$120.49(-26.00,266.97)$

$2.51(-2.44,7.46) \quad-120.73(-392.00,150.54)$

\begin{tabular}{lccccc}
\hline Overall $R^{2}$ & .256 & .077 & .074 & .133 & .040 \\
$F$-test $(10,1933-1940)$ & $66.63^{* *}$ & $16.24^{* *}$ & $15.37^{* *}$ & $29.60^{* *}$ & $8.00^{* *}$ \\
$\Delta R^{2}$ (with Profiles added) & .001 & .005 & $<.001$ & $<.001$ & .001 \\
$\Delta R^{2} F$-test $(3,1933-1940)$ & 0.49 & $3.76^{*}$ & 0.21 & 0.011 & .004 \\
\hline
\end{tabular}

Note. $* p<0.05, * * p<0.01 .{ }^{a}$ Reference category is Highest Education $=$ University Degree. Educ. $=$ Highest Education; ${ }^{b}$ Profiles 1 \& 2 (i.e. Normal

Hearing) is the reference category. HL = Hearing Loss. Denominator degrees of freedom vary due to missing data on some cognitive measures.

Higher scores on all measures indicate better (more accurate or faster) performance. Unless otherwise stated, figures in cells are unstandardized regression coefficients, and figures in parentheses are the bounds of the $95 \%$ confidence intervals. 


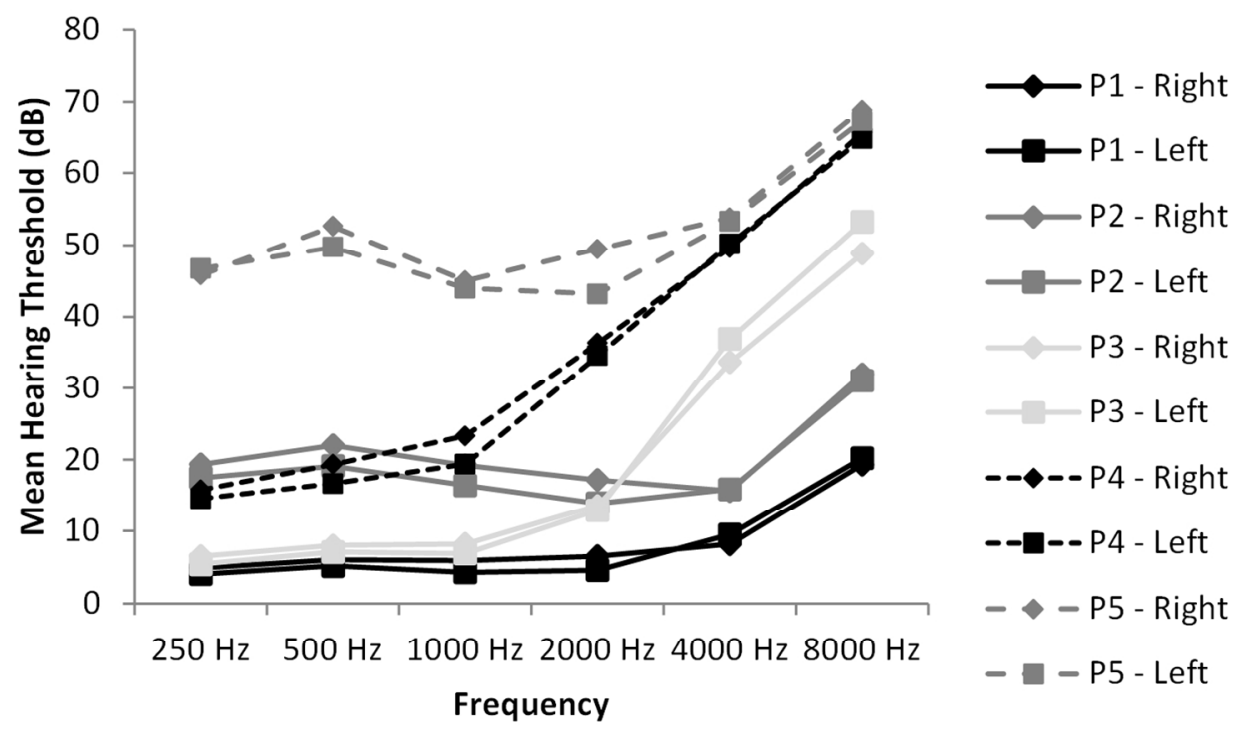

Figure 1.

Hearing thresholds of the left and right ears using a 5-Latent Profile model.

$\mathrm{P}=$ Latent Profile; P1 $\mathrm{n}=1117 ; \mathrm{P} 2 \mathrm{n}=258, \mathrm{P} 3 \mathrm{n}=403, \mathrm{P} 4 \mathrm{n}=153, \mathrm{P} 5 \mathrm{n}=38$.

$127 \times 76 \mathrm{~mm}(300 \times 300$ DPI $)$ 
(Normal Hearing) performed significantly better than members of Profiles 3 and 5, whereas these latter two Profiles could not be separated. For Speed of Memory Retrieval, members of Profile 3 performed less well than members of Profiles $1 \& 2$, and Profile 4.

\section{Discussion}

This study investigated the relationship between hearing impairment and cognitive performance in an epidemiological sample of middle-aged adults, controlling for cognitive reserve, education, age, depression and sex. Contrary to predictions, analysis using a pure tone average measure for the better ear revealed that hearing loss was not a significant predictor of cognitive performance in any domain. With the large sample size, the failure to find a relationship could not be due to insufficient power.

Further, Latent Profile Analysis, which produced four discernible hearing groups, revealed an effect of hearing impairment on continuity of attention, which was generally lower in those with poorer hearing relative to those with normal hearing, and in speed of retrieval of episodic (or long-term) memory (in those with high tone hearing loss only). Nonetheless, the amount of variance in performance explained by hearing profile membership was very small $(\leq 0.5 \%)$, and unlikely to be of clinical significance. The observed results are not consistent with the idea that cognitive performance is meaningfully associated with the extent of hearing impairment.

These findings stand in marked contrast to studies that report hearing impairment is associated with poorer cognition ${ }^{6}$ or is risk factor for cognitive decline ${ }^{9}$ and dementia ${ }^{10,11}$. One possibility is that this study successfully controlled for important covariates not previously considered, or not considered together. In particular, no previous studies controlled for depression or cognitive reserve: critical components of any evaluation of the impact of hearing on cognition. Alternatively, this sample, being middle-aged, may not have progressed 
to levels of hearing impairment sufficient to interfere with cognition. This argument would be supported by the fact that none experienced profound hearing impairment ( $>81 \mathrm{~dB}$ PTA better ear). However, the prevalence of profound hearing impairment is generally low at $0.7 \%{ }^{35}$ and studies with older participants (e.g. ${ }^{6}$ mean 71years) have reported similarly low rates of severe or profound HL, yet found a relationship with cognition. If the cognitive burden of hearing impairment is yet to develop in this cohort, then longitudinal follow-up of this wellcharacterised group, assessing both their hearing impairment, hearing rehabilitation and cognitive performance will be important. The BHAS is longitudinal, making such analyses possible in the future.

This study asked individuals to wear their prescribed hearing aid(s) for cognitive testing. However, not all hearing-impaired people have assistive devices or use them optimally. A study that tests individuals before and after hearing intervention, and which controls for depression, premorbid-IQ, age, education and sex, would help to unpack this issue. Preferably, such a study would also test non-intervention controls to assess the impact of cognitive practice effects.

The method used for analysing hearing level has implications for the hearing loss field. Latent Profile Analysis suggested four discernible groups, with the largest having normal hearing, then high frequency, mid-to-high frequency, and significant hearing impairment. Ciletti and Flamme ${ }^{34}$ found similar profiles in 20 to 69 year olds, using k-means cluster analysis, but also noted marked sex differences, which we did not observe.

Comparison of latent profile analysis with the better-ear PTA classification used by others (e.g. ${ }^{6}$ ) revealed considerable inconsistency. Participants with high-frequency hearing loss were categorised by the better-ear method as having normal hearing, even when their hearing loss extended down to $2000 \mathrm{~Hz}$. Further, 1 in 4 of those in the severely impaired profile were classified as normal on the better-ear measure. This inconsistency challenges definitions of 
significant hearing loss: 403 participants were identified with a significant, high-frequency hearing loss in this study (average hearing loss $\geq 40 \mathrm{~dB}$ at 4 and $8 \mathrm{kHz}$ in the better ear), but all would have been classed as normal according to traditional definitions. These participants could be expected to experience a range of hearing-related social impacts but, as they are classified as normal under the standard system, would have limited access to hearing interventions. This study supports the view that such standard classifications should be used with caution ${ }^{36}$, as they do not capture the impairments associated with high-frequency hearing losses and higher-order auditory processing skills.

\section{Conclusion}

In a large, randomly sampled, representative group of middle-aged adults, hearing loss is not an important predictor of attention, memory or executive function once education, depression, cognitive reserve, and sex have been considered. One possibility is that hearing impairment is just one type of morbidity that, combined with others, builds a risk-profile that would predict cognitive decline in normal ageing. Other important determinants of cognitive performance include chronic health conditions such as smoking ${ }^{37}$, pain ${ }^{38}$, diabetes ${ }^{39}$, or hypertension ${ }^{37,40}$, of which smoking ${ }^{41}$ and diabetes ${ }^{42}$ are also associated with hearing loss. Most studies, this one included, have considered a single morbidity and its impact on cognition. However, as many as 3 in 10 adults aged 45-64 years have 5 or more long-term comorbidities, of which hearing impairment may be just one ${ }^{43}$. If the risk of cognitive decline arises additively then we should stop trying to isolate any single cause. The BHAS, with its longitudinal follow-up, is ideally placed to inform this important debate.

Acknowledgements: We thank the operational team of the Busselton Healthy Ageing Study based in Busselton of Elspeth Inglis, Jenifer George, Jessica Storey, Aida Embling, Debra 
Burwood, Dianne Toovey, Stephanie Murphy, Darcy Bennett and Shelley Cheetham for participant recruitment and data collection, and the community of Busselton for their ongoing support and participation. We thank the Western Australian Country Health Service - South West for core infrastructure support and Bracket Global for the CDR System computerised assessment system. 


\section{References}

1. WHO. Millions of people in the world have hearing loss thay can be treated or prevented. Periodical [serial online]. Date 2013. Available from: World Health Organisation, Geneva.

2. Cruickshanks KJ, Wiley TL, Tweed TSet al. Prevalence of hearing loss in older adults in Beaver Dam, Wisconsin. The Epidemiology of Hearing Loss Study. Am J Epidemiol 1998; 148:879-886.

3. Gopinath B, Schneider J, McMahon CM, Teber E, Leeder SR, Mitchell P. Severity of age-related hearing loss is associated with impaired activities of daily living. Age Ageing 2012; 41:195-200.

4. Hua H, Karlsson J, Widen S, Moller C, Lyxell B. Quality of life, effort and disturbance perceived in noise: a comparison between employees with aided hearing impairment and normal hearing. Int J Audiol 2013; 52:642-649.

5. Preminger JE, Meeks S. The influence of mood on the perception of hearing-loss related quality of life in people with hearing loss and their significant others. Int J Audiol 2010; 49:263-271.

6. Lin FR, Ferrucci L, Metter EJ, An Y, Zonderman AB, Resnick SM. Hearing loss and cognition in the Baltimore Longitudinal Study of Aging. Neuropsychology 2011; 25:763-770.

7. Lindenberger U, Baltes PB. Sensory functioning and intelligence in old age: a strong connection. Psychol Aging 1994; 9:339-355.

8. Tay T, Wang JJ, Kifley A, Lindley R, Newall P, Mitchell P. Sensory and cognitive association in older persons: findings from an older Australian population. Gerontology 2006; 52:386-394.

9. Lin FR, Yaffe K, Xia Jet al. Hearing loss and cognitive decline in older adults. JAMA Intern Med 2013; 173:293-299.

10. Gallacher J, Ilubaera V, Ben-Shlomo Yet al. Auditory threshold, phonologic demand, and incident dementia. Neurology 2012; 79:1583-1590.

11. Lin FR, Metter EJ, O'Brien RJ, Resnick SM, Zonderman AB, Ferrucci L. Hearing loss and incident dementia. Arch Neurol 2011; 68:214-220.

12. Tun PA, McCoy S, Wingfield A. Aging, hearing acuity, and the attentional costs of effortful listening. Psychol Aging 2009; 24:761-766.

13. Wingfield A, Tun PA, McCoy SL. Hearing Loss in Older Adulthood - What It Is and How It Interacts With Cognitive Performance. Curr Dir Psychol Sci 2005; 14:144148.

14. Barnes LL, Mendes de Leon CF, Wilson RS, Bienias JL, Evans DA. Social resources and cognitive decline in a population of older African Americans and whites. Neurology 2004; 63:2322-2326.

15. Bennett DA, Schneider JA, Tang Y, Arnold SE, Wilson RS. The effect of social networks on the relation between Alzheimer's disease pathology and level of cognitive function in old people: a longitudinal cohort study. Lancet Neurol 2006; 5:406-412.

16. Arlinger S. Negative consequences of uncorrected hearing loss--a review. Int J Audiol 2003; 42 Suppl 2:2S17-20.

17. Lee RS, Hermens DF, Porter MA, Redoblado-Hodge MA. A meta-analysis of cognitive deficits in first-episode Major Depressive Disorder. J Affect Disord 2012; 140:113-124. 
18. La Rue A. Healthy brain aging: role of cognitive reserve, cognitive stimulation, and cognitive exercises. Clin Geriatr Med 2010; 26:99-111.

19. Singh-Manoux A, Kivimaki M, Glymour MMet al. Timing of onset of cognitive decline: results from Whitehall II prospective cohort study. BMJ 2012; 344:d7622.

20. Mangialasche F, Kivipelto M, Solomon A, Fratiglioni L. Dementia prevention: current epidemiological evidence and future perspective. Alzheimers Res Ther 2012; 4:6.

21. Kroenke K, Spitzer RL, Williams JB. The PHQ-9: validity of a brief depression severity measure. J Gen Intern Med 2001; 16:606-613.

22. James A, Hunter M, Straker Let al. Rationale, design and methods for a community-based study of clustering and cumulative effects of chronic disease processes and their effects on ageing: the Busselton healthy ageing study. BMC Public Health 2013; 13:936.

23. Margolis RH, Saly GL, Le C, Laurence J. Qualind: A method for assessing the accuracy of automated tests. J Am Acad Audiol 2007; 18:78-89.

24. Eikelboom RH, Swanepoel D, Motakef S, Upson GS. Clinical validation of the AMTAS automated audiometer. Int J Audiol 2013; 52:342-349.

25. Lazarsfeld PF, Henry NW. Latent structure analysis. New York: Houghton Mifflin, 1968.

26. Keith MS, Stanislav SW, Wesnes KA. Validity of a cognitive computerized assessment system in brain-injured patients. Brain Inj 1998;

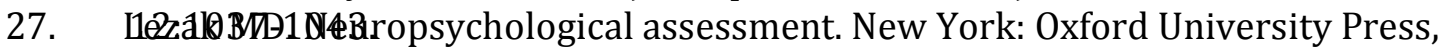
1995.

28. Nelson HE, Willison JR. National adult reading test (NART): Test manual (2nd ed.). Windsor, UK: NFER-Nelson, 1991.

29. McGurn B, Starr JM, Topfer JAet al. Pronunciation of irregular words is preserved in dementia, validating premorbid IQ estimation. Neurology 2004; 62:1184-1186.

30. Wesnes KA, Ward T, McGinty A, Petrini O. The memory enhancing effects of a Ginkgo biloba/Panax ginseng combination in healthy middle-aged volunteers. Psychopharmacology (Berl) 2000; 152:353-361.

31. Nylund KL, Asparouhov T, Muthen BO. Deciding on the number of classes in latent class analysis and growth mixture modeling: A Monte Carlo simulation study. Structural Equation Modeling 2007; 14:535-569.

32. Muthén BO, Muthén LK. Integrating person-centered and variable-centered analyses: Growth mixture modeling with latent trajectory classes. Alcoholism: Clinical and Experimental Research 2000; 24:882-891.

33. Muthén LK, Muthén BO. Mplus: Statistical analysis with latent variables: User's Guide Los Angeles, CA: Muthén \& Muthén, 2014.

34. Ciletti L, Flamme GA. Prevalence of hearing impairment by gender and audiometric configuration: results from the National Health and Nutrition Examination Survey (1999-2004) and the Keokuk County Rural Health Study (1994-1998). J Am Acad Audiol 2008; 19:672-685.

35. Turton L, Smith P. Prevalence \& characteristics of severe and profound hearing loss in adults in a UK National Health Service clinic. Int J Audiol 2013; 52:92-97.

36. Clark JG. Uses and abuses of hearing loss classification. ASHA 1981; 23:493-500.

37. Knopman D, Boland LL, Mosley Tet al. Cardiovascular risk factors and cognitive decline in middle-aged adults. Neurology 2001; 56:42-48. 
38. Moriarty O, McGuire BE, Finn DP. The effect of pain on cognitive function: a review of clinical and preclinical research. Prog Neurobiol 2011; 93:385-404.

39. McCrimmon RJ, Ryan CM, Frier BM. Diabetes and cognitive dysfunction. The Lancet 2012; 379:2291-2299.

40. Skoog I. Hypertension and cognition. Int Psychogeriatr 2003; 15:139-146.

41. Cruickshanks KJ, Klein R, Klein BE, Wiley TL, Nondahl DM, Tweed TS. Cigarette smoking and hearing loss: the epidemiology of hearing loss study. JAMA 1998; 279:1715-1719.

42. Bainbridge KE, Hoffman HJ, Cowie CC. Diabetes and hearing impairment in the United States: audiometric evidence from the National Health and Nutrition Examination Survey, 1999 to 2004. Ann Intern Med 2008; 149:1-10.

43. Australian Bureau of Statistics. National health survey 2004-2005: summary of results: Canberra, 2006. 